\title{
Las aspiraciones educativas de la juventud fronteriza: el caso de Tijuana
}

\section{Educational aspirations of the border youth: the case of Tijuana}

Recibido el 16 de mayo de 2021. Aceptado el 16 de febrero de 2022. Publicado el 24 de febrero de 2022.

*Autor para correspondencia: José G. Aguilar Barceló. Correo electrónico: gaba@uabc.edu.mx

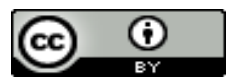

Esta obra está protegida bajo una licencia Creative Commons Atribución 4.0 Internacional.

\author{
José G. Aguilar Barcelóa ${ }^{*}$ (D) https://orcid.org/0000-0002-6378-6886 \\ Germán Osorio-Novela ${ }^{a}$ (i) https://orcid.org/0000-0003-0157-6979 \\ Guillermo A. Aguilar Solís ${ }^{b}$ (D) https://orcid.org/0000-0002-3986-7264 \\ Ana B. Mungaray-Moctezuma ${ }^{a}$ (D) https://orcid.org/0000-0003-3165-8617
}

${ }^{a}$ Universidad Autónoma de Baja California, Facultad de Economía y Relaciones Internacionales. Tijuana, México, correo electrónico: gaba@uabc.edu.mx, gosorio@uabc.edu.mx, bmungaray@uabc.edu.mx

${ }^{\mathrm{b}}$ El Colegio de la Frontera Norte, estudiante de doctorado en estudios de migración, Tijuana, México, correo electrónico: guillermo.agsolis@gmail.com

\section{Resumen}

El presente artículo pretende identificar la influencia del contexto transfronterizo en las aspiraciones educativas de los estudiantes de educación media superior que radican en Tijuana, Baja California. Para este objetivo se estiman modelos de regresión de respuesta cualitativa que consideran variables de índole demográfica y socioeducativa, del ámbito migratorio, y relacionadas con el empeño, la motivación propia y el apoyo familiar. Se utilizan datos recopilados por el Mexican Migration Field Research Program. Se encontró que las expectativas son poco sensibles a las circunstancias migratorias y transfronterizas. En cambio, el nivel educativo de los padres, la dedicación al estudio, tener familiares cercanos estudiando y saber inglés son variables que sí parecen relevantes en la formación de las expectativas. Se concluye que los aspectos demográficos y socioeducativos, así como la dedicación propia y el apoyo familiar, determinan en mayor medida las aspiraciones de los jóvenes.

Palabras clave: jóvenes, comunidades transfronterizas, Tijuana, aspiraciones académicas, educación media superior, educación superior.

\section{Abstract}

This article aims to shed light on the impact of the cross-border context on educational aspirations of middle and high school students living in Tijuana, Baja California. For this purpose, qualitative response regression models are estimated considering variables of a demographic and socio-educational nature, of the migratory field and related to commitment, self-motivation, and family support. Data was collected by the Mexican Migration Field Research Program is used. We found that

CÓMO CITAR: Aguilar Barceló, J. G., Osorio-Novela, G., Aguilar Solís, G. A. \& Mungaray-Moctezuma, A. B. (2022). Las aspiraciones educativas de la juventud fronteriza: el caso de Tijuana [Educational aspirations of the border youth: the case of Tijuana]. Estudios Fronterizos, 23, e087. https://doi.org/10.21670/ref.2203087 
expectations are not very sensitive to migratory and cross-border circumstances. On the other hand, the educational level of the parents, dedication to study, have family members at the university and speak English, does seem relevant in the formation of expectations. We concluded that demographic and socio-educational factors, as well as their own effort, and family support determine to a greater extent the aspirations of the youth.

Keywords: youth, cross-border communities, Tijuana, academic aspirations, high school, higher education.

\section{Introducción}

Si bien la escolaridad de las personas depende en gran medida de sus características socioeconómicas (Espitia Carrascal \& Montes Rotela, 2009), cabe esperar también que las dinámicas familiares y laborales influyan en la concepción acerca del desarrollo profesional y, con ello, del valor de lo académico (Orraca et al., 2017). Al tener en cuenta que las familias de la frontera entre México y Estados Unidos (EuA) viven en entornos multiculturales asociados a asimetrías laborales, separaciones y reencuentros familiares y experiencias que producen cambios en la conducta social de la población, en las últimas décadas se ha intensificado el estudio de los efectos, tanto positivos como negativos, que el fenómeno migratorio y transfronterizo entre estos dos países puede llegar a tener en las aspiraciones educativas de los jóvenes y su influencia en las decisiones individuales en materia de formación.

Entre los efectos positivos encontrados en la literatura suelen destacar aquellos de naturaleza económica que resultan del fomento de las actividades productivas en la comunidad de destino ${ }^{1}$ (Lindstrom, 1996; Massey \& Parrado, 1998), pero también en la de origen, a través del envío de remesas (Giorguli \& Serratos, 2009). Este tipo de efectos también podrían estar representados por una mayor inversión en capital humano en el mediano plazo (principalmente en educación y salud). El trabajo transfronterizo, por otro lado, permite optar a mejores salarios en términos relativos. Por último, siempre cabe la posibilidad de que los migrantes de retorno ${ }^{2}$ se conviertan en agentes de cambio y difusores de ideas novedosas que puedan ser implementadas en los lugares de origen, favoreciendo así la organización productiva y social de la comunidad (Goldscheider, 1987; Mendoza Cota, 2013).

Por otra parte, dentro de los efectos negativos están los costos sociales y emocionales de una potencial disrupción de la convivencia o de la separación de las familias. A este respecto, Antman (2011) sugiere que las personas menores de edad reducen su dedicación al estudio y participación en actividades escolares cuando hay una separación transfronteriza de los padres. Además, las propias remesas podrían llegar a ser causa de abandono escolar y de retraso en el desarrollo de la comunidad de origen (Giorguli \& Serratos, 2009) al fomentar sistemas locales de producción no

\footnotetext{
${ }_{1}^{1}$ Para McConnell y colaboradores (2007) la migración laboral mejora el funcionamiento de los mercados de trabajo al fomentar la eficiencia asignativa.

${ }^{2}$ Los migrantes de retorno son aquellos individuos que regresan a su país de origen, de forma voluntaria o inducida, con el fin de permanecer en él, después de haber radicado en otra nación (Izquierdo Escribano, 2011).
} 
autosostenibles, que socavan los recursos humanos en edad escolar o laboral y frenan el cambio en la estructura productiva (Fischer et al., 1997).

En la zona geográfica que conforma la frontera entre México y euA se produce la confluencia de diversos y heterogéneos grupos sociales que interactúan para realizar actividades laborales, turísticas, artísticas y médicas, entre otras. Durante muchos años estas actividades han logrado dar empuje a la economía fronteriza al incrementar el empleo y, en consecuencia, atraer a la población inmigrante procedente del sur. Sin embargo, el mercado laboral de esta región ha venido perdiendo capacidad de absorción, a la vez que se ha dado una sobredemanda de infraestructura pública, lo que se manifiesta, por ejemplo, en la precarización laboral (Cruz Piñeiro, 2012) y el rezago educativo. ${ }^{3}$

En relación con esto último, trabajos como los de Coubès y González Ramírez (2011) y Vargas-Valle (2012) documentan bajos niveles de escolarización en las ciudades fronterizas, en comparación con los del resto del país, debido a la alta migración interna y a la dinámica del mercado laboral fronterizo. De acuerdo con Kandel y Massey (2002), las personas menores de edad que ven con naturalidad la migración laboral internacional tienen hasta cinco veces más probabilidades de emigrar que aquellos que son ajenos a esa circunstancia. Asimismo, en algunas zonas de México, los estudiantes de educación media superior tienen el doble de probabilidad de buscar trabajo en EuA que de continuar su formación académica. Las realidades anteriores podrían tener consecuencias en la equidad educativa de zonas como la frontera.

La educación es una vía de revalorización del conocimiento y de obtención de formación y experiencias que permiten ser más productivo profesionalmente (Münch, 2005), y optar a mejores oportunidades laborales y salarios más altos (Leyva López \& Cárdenas Almagro, 2002). Los gastos en educación y capacitación son inversiones en capital humano, pero también lo son aquellos realizados en materia de salud, el conocimiento sobre el mercado de trabajo o la crianza de la prole (McConnell et al., 2007). Para Becker (1964), la educación incrementa las ganancias de las personas en la medida en que el entorno en el que se desempeñan esté menos desarrollado.

Aunque en México la educación superior es entendida como el principal factor de transformación social ${ }^{4}$ (Marmolejo \& León, 2000), en materia laboral se encuentra que dos terceras partes de la población adulta joven de Tijuana se desempeña como empleada u obrera. La elevada concentración en esta categoría ocupacional se vincula con la presencia de la industria maquiladora en la región. Son losjóvenes, precisamente, los que tienen mayor participación en esta forma de organización industrial (González Galbán, 2011). La facilidad para encontrar trabajo asalariado de baja calificación, junto con el arraigo de la cultura de la migración, podrían hacer a estas alternativas más atractivas para lograr la movilidad social o para alcanzar el estándar de vida deseado, que la dedicación a los estudios (Meza González \& Pederzini Villarreal, 2009). Cabe destacar que, según datos del Instituto Nacional de Estadística y Geografía (Inegi, 2015), 52\% de los jóvenes de Tijuana forman parte de la población económicamente activa; cuatro puntos porcentuales por encima de la media nacional.

El presente trabajo pretende arrojar luz sobre el efecto que la exposición a las dinámicas demográfica y socioeducativa de la frontera, el entorno migratorio y

\footnotetext{
${ }^{3}$ Según datos de 2015 del Instituto Nacional para la Educación de los Adultos en http://www.inea.gob.mx/ transparencia/pdf/rez_censo_edos/rez_ei15_mun_ur_02.pdf

${ }^{4}$ A diferencia de lo que ocurre en EUA, donde frecuentemente los servicios educativos se consideran bienes de consumo.
} 
los anhelos profesionales y soporte familiar tienen en las aspiraciones educativas y profesionales de los estudiantes de educación media superior residentes del lado mexicano de la zona fronteriza entre Tijuana, en Baja California, y San Diego, en California, la cual ha sido calificada como una de las más dinámicas del mundo (Ojeda, 2009). Se parte de las vulnerabilidades que estos niveles educativos presentan para plantear la hipótesis de que las aspiraciones de los jóvenes que los cursan son más sensibles a la exposición al entorno migratorio que al empeño, la motivación y el respaldo recibido por la familia, y que la asociación entre estas aspiraciones y la exposición al entorno migratorio es negativa.

\section{Marco referencial}

\section{El contexto migratorio y transfronterizo de Tijuana}

La dinámica fronteriza entre México y EUA no podría entenderse cabalmente sin tomar en cuenta el fenómeno migratorio. En la actualidad, el flujo de migrantes mexicanos a EUA es muy diferente al de hace algunas décadas cuando se componía principalmente de jóvenes varones en búsqueda de trabajo agrícola. Durante los últimos años, otros muchos colectivos han participado en este proceso con fines diversos, lo que lo convierte en un fenómeno mucho más complejo que antaño.

Hay dos procesos demográfico-migratorios — contemporáneos y coyunturalesque están relacionados con la frontera septentrional mexicana y que deben ser tomados en cuenta en virtud de que dan forma a la identidad cultural de las sociedades que los experimentan. El primero está asociado al incremento en el número de inmigrantes internacionales retornantes a los municipios fronterizos de México desde 2017 (Inegi, 2020; Masferrer \& Roberts, 2012). La frontera ha dejado de ser solo el imán de la migración interna, para convertirse también en uno de migración de retorno a partir del segundo lustro del presente siglo (Canales, 2012; Vargas Valle, 2015), debido en parte a la burbuja inmobiliaria que estalló en 2008 en EuA que llevó a una crisis financiera global, ${ }^{5}$ reforzada por el surgimiento de un clima antiinmigrante en aquel país durante la administración del presidente Donald Trump.

El segundo proceso tiene que ver con la inmigración a Tijuana procedente de otras partes del país, además de la referida a Centroamérica, Sudamérica y el Caribe, a través de la cual se explica, en buena medida, el crecimiento histórico de la ciudad y por la que esta goza de una alta multiculturalidad. Sobre el particular destaca la crisis migratoria acaecida en 2016, por la que habrían llegado cerca de 3000 haitianos a Tijuana; asimismo, durante 2018 se registró en la ciudad la llegada de más de 6000 inmigrantes centroamericanos con la intención de pedir asilo en EUA $^{6}$ (El Colegio de la Frontera Norte, 2019). Al respecto, datos de la encuesta intercensal del Inegi (2015) indican que la proporción de la población no nativa de Tijuana en 2015 era de $47 \%$.

${ }^{5}$ El desequilibrio económico que se dio en EUA a partir de 2008 trajo consigo el incremento del desempleo a niveles que no se observaban desde la década de 1980. Para Calderón y colaboradores (2010), esto potenció la pobreza, la desigualdad, la informalidad y la aglomeración desordenada en la frontera.

${ }^{6}$ Aunque muchas veces, por razones diversas, estos migrantes terminan por radicarse en la ciudad. 
Kandel y Massey (2002) argumentan que, en algunas partes de México, la migración hacia EUA es vista como un elemento de transición hacia la madurez, por lo que la acción es aprobada socialmente y quien lo hace es reconocido como persona responsable y visionaria. Este anhelo se contagia a través del entorno social y por la observación del papel que juegan aquellos que lo han hecho, quienes, al regresar, suelen mostrar un cambio en sus circunstancias económicas, lo que lleva al fortalecimiento de la conjetura en los jóvenes de que la inversión en educación no es tan redituable debido a la falta de calificación de los puestos que posiblemente ocuparán, lo que hace que estos reduzcan sus esfuerzos académicos. Ellos encuentran que la aspiración de residencia en EuA generalmente no está relacionada con el anhelo de los padres sobre la continuidad educativa, aunque destacan que, en los alumnos de nivel medio superior, la intención de trabajar allende la frontera sí es inversa al interés de seguir estudiando en México.

Las acciones mediante las cuales los migrantes llegan a forjar y mantener relaciones sociales simultáneas entre su sociedad de origen y la de asentamiento (Glick Schiller et al., 1995) son una manifestación de la "transnacionalidad"7 (Zúñiga \& Reyes, 2006). Muchas veces, estos migrantes se afincan en zonas próximas a la frontera, ya sea para no perder los lazos afectivos con el país de procedencia, o por contar ya con familiares o amigos en esas zonas o, en muchas ocasiones, por limitaciones presupuestarias. ${ }^{8}$ Cuando la vida cotidiana de estas familias o comunidades dependen de interconexiones constantes a través de una frontera internacional y sus integrantes se incorporan en la economía, patrones de vida cotidianos e instituciones políticas de ambos países marcan su identidad pública (Glick Schiller et al., 1992) con lo que se está ante la presencia de familias o comunidades transfronterizas.

\section{Migración y educación: algunos resultados teóricos y empíricos}

Para Vargas Valle (2015) existen fuerzas encontradas en la relación entre migración y educación, cuyo efecto conjunto en la población más joven aun es incierto. Por una parte, la migración podría generar ganancias económicas que posibiliten la dedicación exclusiva a los estudios (Taylor, 1987), pero, por la otra, podría llevar el capital social del hogar a un umbral por debajo de aquel que garantice el éxito escolar (Booth, 1995). Asimismo, cabe la posibilidad de que el fenómeno tenga un efecto negativo en las aspiraciones educativas de los jóvenes, cuando en el hogar se sobrevaloran los beneficios económicos y culturales del trabajo en EUA (Kandel \& Massey, 2002). Para Vargas-Valle y Glick (2021) las aspiraciones educativas y migratorias de los estudiantes de educación media superior en Tijuana son sensibles a la experiencia trasfronteriza

\footnotetext{
${ }^{7} \mathrm{Al}$ respecto, las familias transnacionales tienen presencia simultánea en dos países a través del flujo migratorio internacional (generalmente alternado) de sus miembros. Estas familias, a diferencia de las transfronterizas, pueden estar sujetas a condiciones sociales muy distintas según sea su localización y el estatus migratorio de sus miembros dado que no se localizan necesariamente en comunidades contiguas entre sí (Ojeda, 2009). En este sentido, el nexo transfronterizo podría considerarse una forma de transnacionalidad.

${ }^{8}$ Es importante señalar que la condición de migrante no supone la manifestación de la transnacionalidad, aunque el transnacionalismo sí tiene como base algún tipo de experiencia migratoria. De cualquier forma, haber nacido en EUA y radicar en México no es condición suficiente para ser considerado migrante.
} 
y al nivel de capital social que la vinculación con EuA represente en términos de ciudadanía, familiares, dominio del inglés o identificación cultural. También, los estudiantes con educación y otros vínculos transfronterizos, bajo ciertas circunstancias, tienen más probabilidades que el resto de aspirar a un título universitario, pero también de migrar o de participar en un empleo al otro lado de la frontera. ${ }^{9}$

En ciudades fronterizas como Tijuana, consideradas como receptoras del turismo y corrientes migratorias, la estructura educativa evoluciona con la de fenómenos geopolíticos y económicos. Mientras que en 2008 la proporción de estudiantes en educación básica, media superior y superior era, respectivamente, de 47\%, 38\% y $15 \%$ (Inegi, 2010), para 2018 estos indicadores habían cambiado a 69\%, 17\% y 14\% (Sistema Educativo Estatal de Baja California [SEEBC], 2018); ya para 2021 se situaban en $71 \%$, $15 \%$ y $14 \% .{ }^{10}$ Aunque esto no permite asegurar que la educación media superior sea el nivel más sensible al entorno, sí es indicio de que este nivel educativo presenta el mayor reto de atención con miras a incrementar la retención en los próximos años, no solo por su magnitud actual y su importante descenso en los últimos años, sino por el grado de concentración del nivel precedente, que llegaba a 71\% en 2021.

Estudios como los de Hanson y Woodruff (2003) y Borraz (2005) sugieren un potencial efecto positivo de la migración sobre la educación de los más jóvenes en los casos de baja escolaridad de la madre y hogares donde se reciben remesas, aunque sus resultados se enfocan en comunidades rurales. $\mathrm{Al}$ respecto, para Nobles (2011), los lazos con los padres migrantes se correlacionan positivamente con los resultados escolares, lo que mitiga los costos educativos de una separación familiar. Por su parte, Antman (2011) encuentra que los menores de edad con padre migrante asignan menos tiempo a la realización de tareas escolares ante la falta de un patrón de conducta masculino. Lo anterior se debe a que las aspiraciones del menor terminarían estando relacionadas con la migración laboral o la percepción de falta de apoyo, lo que genera un costo psicológico. La autora concluye que los efectos negativos de la migración paterna podrían ser superiores a los beneficios que se pueden alcanzar a través de las remesas.

Por su parte, McKenzie y Rapoport (2011) encuentran un efecto negativo de la migración en el logro académico de los adolescentes de zonas rurales que podría reducir las posibilidades de que se complete la educación media en hasta $22 \%$, aunque este efecto se mitiga con las remesas que relajan las restricciones sobre la inversión en educación. En este sentido, Kandel y Kao (2000) encuentran que las remesas relajan lo suficiente las presiones económicas de los hogares que las reciben como para que las personas menores de edad tengan una mayor dedicación al estudio, pero solo hasta que estas personas adquieren autonomía en la toma de decisiones durante la adolescencia, que es cuando pueden ser más fuertemente influenciados por la actividad ocupacional y educativa de sus familiares y conocidos. Sus resultados muestran también que los jóvenes con mejores calificaciones y aquellos cuyos padres cuentan con una ocupación profesional tienen menos interés en buscar trabajo fuera

\footnotetext{
${ }^{9}$ En muchos de estos casos la persona (generalmente llamada transmigrante o commuter) trabaja en EUA, pero vive del lado mexicano.

${ }^{10}$ Datos tomados de la estadística educativa de Baja California para el ciclo escolar 2020-2021 publicada por la Secretaría de Educación Pública en https://planeacion.sep.gob.mx/Doc/estadistica_e_indicadores/ estadistica_e_indicadores_entidad_federativa/estadistica_e_indicadores_educativos_02BC.pdf
} 
del país; sin embargo, cuando los estudiantes han repetido algún grado escolar, el interés aumenta.

Por su parte, Coubès y González Ramírez (2011) estudian la interrelación entre la escuela y el trabajo para la población joven de Tijuana y encuentran que los nacidos fuera del estado de Baja California, en mayor proporción que los locales, combinan el estudio con el trabajo o trabajan de forma exclusiva. También, identifican que solo $48 \%$ de los que completan la educación básica alcanzan el nivel medio superior. Los autores afirman que el mercado laboral de la juventud tijuanense es más accesible que el del resto de México, pero también de más baja calificación.

Por otro lado, Vargas Valle (2015) analiza la continuidad escolar de los jóvenes de áreas urbanas de la frontera norte de México con dos características: haber nacido en EUA y compartir hogar con personas radicadas en México que trabajan en EUA. La autora encuentra que el país de nacimiento tiene mayor asociación con el nivel de educación alcanzado, que el hecho de tener familiares con las características descritas. También, los jóvenes que residen con alguna persona con esta actividad transfronteriza podrían llegar a presentar ventajas en la conclusión de la educación básica y media superior, pero no en el caso de la universidad.

Los estudiantes radicados en México pero que nacieron y estudian en EuA aumentan su capital humano - específico de EUA-y, con ello, la probabilidad de poder encontrar un empleo bien remunerado al norte de la frontera (Orraca et al., 2017). Otro tipo de vinculación transfronteriza ocurre para aquellos que, aun habiendo nacido en México, cruzan frecuentemente la frontera internacional con fines turísticos, de negocios o de salud, apoyados en sus altos niveles de ingresos y estando constituidos en gran medida por lo que Sarabia (2015) denomina cosmopolitas del Sur Global, que es una clase privilegiada y móvil, compuesta por miembros de clase media y alta. Estas características hacen que los hogares con nexos transfronterizos no sean del todo comparables con los de los inmigrantes (Orraca et al., 2017).

La Tabla 1 muestra las diferencias en importancia que dan los jóvenes a la educación superior en ambos lados de la frontera, las cuales se comentan a la luz de la perspectiva transversal transfronteriza del lado mexicano.

De acuerdo con la Tabla 1, el porcentaje de jóvenes que le da alta importancia a la asistencia a la universidad y que está muy de acuerdo en que la dedicación a la escuela es una vía para alcanzar un mejor futuro laboral es mayor en Tijuana que en San Diego. Una diferencia significativa entre los dos conjuntos poblacionales ocurre ante la afirmación de que las clases tomadas en el nivel medio superior contribuyen en la construcción del futuro, pues mientras que en San Diego hasta $35 \%$ de los estudiantes está en desacuerdo o muy en desacuerdo, esto ocurre solo en $14 \%$ de los casos del lado mexicano. También, hay una discrepancia notable en la opinión de los estudiantes en relación con el deseo de los padres de que asistan a la universidad, afirmación con la que está muy de acuerdo $61 \%$ en Tijuana frente a $49 \%$ en San Diego. Se debe destacar que ambas localidades coinciden en la ordenación jerárquica de sus respuestas y que no hubo diferencia estadística cuando se les preguntó acerca del papel que jugaba la universidad en la consecución de un buen trabajo. Por otro lado, la variabilidad en las respuestas (equivalente al grado de radicalización) siempre fue mayor en el caso de Tijuana. 
Tabla 1. Percepción de la educación superior de los jóvenes residentes de Tijuana y San Diego (porcentajes) ${ }^{11}$

\begin{tabular}{|c|c|c|c|c|}
\hline Variable & $\begin{array}{c}\text { Respuestas } \\
\text { posibles }\end{array}$ & Tijuana (\%) & San Diego (\%) & $\begin{array}{l}p \text { - valor del } \\
\text { estadístico }\end{array}$ \\
\hline \multirow{3}{*}{ Asistir a la universidad es... } & Muy importante & 65.85 & 59.85 & \multirow{3}{*}{0.000} \\
\hline & Importante & 28.07 & 32.37 & \\
\hline & Poco importante & 6.08 & 7.78 & \\
\hline \multirow{4}{*}{$\begin{array}{l}\text { Trabajar duro en la escuela } \\
\text { lleva a una carrera exitosa }\end{array}$} & Muy de acuerdo & 45.50 & 39.57 & \multirow{4}{*}{0.002} \\
\hline & De acuerdo & 46.21 & 50.87 & \\
\hline & En desacuerdo & 6.08 & 6.81 & \\
\hline & Muy en desacuerdo & 2.21 & 2.75 & \\
\hline \multirow{4}{*}{$\begin{array}{l}\text { Mis clases me preparan } \\
\text { para lo que quiero ser en el } \\
\text { futuro }\end{array}$} & Muy de acuerdo & 34.19 & 16.76 & \multirow{4}{*}{0.000} \\
\hline & De acuerdo & 51.42 & 48.74 & \\
\hline & En desacuerdo & 11.13 & 25.76 & \\
\hline & Muy en desacuerdo & 3.26 & 8.74 & \\
\hline \multirow{4}{*}{$\begin{array}{l}\text { Mis padres quieren que } \\
\text { vaya a la universidad }\end{array}$} & Muy de acuerdo & 60.95 & 49.42 & \multirow{4}{*}{0.000} \\
\hline & De acuerdo & 33.32 & 43.04 & \\
\hline & En desacuerdo & 3.35 & 5.75 & \\
\hline & Muy en desacuerdo & 2.38 & 1.79 & \\
\hline \multicolumn{2}{|c|}{ Número de observaciones } & 2004 & 2070 & \\
\hline
\end{tabular}

Fuente: Elaboración propia con datos del MMFRP 2015-2016 y con base en Aguilar Barceló y colaboradores (2020).

Nota: Los porcentajes corresponden a la frecuencia de cada respuesta en relación al total por variable.

La mayor importancia de la educación superior en las percepciones de los jóvenes de Tijuana podría tener relación con lo advertido por Marmolejo y León (2000), en el sentido de que, en México, este nivel educativo es visto como la base de un proceso de trasformación social y de superación personal con fuertes componentes de aspiraciones, mientras que en EuA es percibido como una inversión (no accesible para todos) que incrementa la probabilidad de obtener un mejor ingreso en el futuro.

\section{Metodología}

\section{Fuentes de información}

Los datos utilizados provienen del Mexican Migration Field Research Program (MMFRP) 2015-2016 de la Universidad de California en San Diego en colaboración con El Colegio de la Frontera Norte y la Universidad Autónoma de Baja California. ${ }^{12}$

\footnotetext{
${ }^{11}$ La última columna de la Tabla 1 y de la Tabla 3 muestra el resultado de la prueba chi-cuadrado de independencia para cada variable. En estas tablas se omiten las variables que resultaron no significativas.

${ }^{12} \mathrm{Al}$ haberse tratado de una encuesta especial dentro del programa, no se cuenta con datos comparables más recientes.
} 
Se contó con una muestra de 4074 estudiantes de educación media superior de entre 14 y 17 años, inscritos en 63 escuelas de Tijuana y San Diego. De estos, 2004 son estudiantes radicados en Tijuana, dentro de los que se identificó a 207 con experiencia o vínculos transfronterizos que, para efectos de este trabajo, están dados por el hecho de haber estudiado, vivido o nacido en EUA o tener o haber tenido un familiar nuclear radicando en este país. Los participantes fueron seleccionados por muestreo aleatorio ponderado en dos etapas: primero por escuela y luego por aula. Cabe señalar que, aunque este trabajo se enfoca en los jóvenes de Tijuana, para algunos indicadores se hacen comparaciones someras con sus similares de San Diego.

La muestra del presente estudio permite afirmar que 5\% de los jóvenes entre 14 y 17 años radicados en Tijuana había nacido en el vecino país del norte, mientras que esto había ocurrido en $39 \%$ del subconjunto con vinculación o experiencia transnacional. Había tenido alguna experiencia académica en ese país $5 \%$ y $8 \%$ había vivido ahí por lo menos un mes. De acuerdo con Rocha Romero y Orraca Romano (2018), la mayoría de los estudiantes que radican en México y estudian en Estados Unidos nacieron en EUA, tanto en el nivel educativo básico (85.1\%), como en el medio superior (74.6\%) y el superior $(64.8 \%)$, pero que, sin embargo, la mayoría de los residentes en México nacidos en EUA no estudia al otro lado de la frontera al encontrar algún tipo de obstáculo con los costos de la educación, los ingresos familiares, el dominio del inglés, el aprovechamiento escolar o incluso el fatigante cruce continuo. ${ }^{13}$

\section{Modelo de regresión de respuesta cualitativa}

La estimación de modelos donde la variable dependiente es cualitativa usualmente tiene como objetivo encontrar la probabilidad de que un determinado evento suceda. En este caso, se parte de un modelo de regresión lineal simple representado por:

$$
Y=\alpha+\beta_{i} X_{i}+u
$$

donde, $Y$ es una variable de respuesta categórica, que, para efectos de cuantificación, se asocia a los valores 0 y 1 , según los intereses del modelo. Además, $\alpha$ es el intercepto, $\beta_{i}$ $(1, \ldots, n)$ son los coeficientes estimados de las variables independientes o explicativas, nombradas $X$, que marcan la magnitud de afectación a la variable dependiente, y $u$ es la perturbación o término de error. La expresión 1 puede ser interpretada en términos probabilísticos como un modelo de elección discreta (Gujarati \& Porter, 2010). No obstante, dado que no hay garantía de que al obtener $\hat{Y}$ por mínimos cuadrados ordinarios, los estimadores de la probabilidad de que ocurra el suceso $Y$ dado $\mathrm{X}$, cumplan con la condición planteada, es conveniente transformar el modelo de tal forma que las predicciones caigan en el intervalo [0,1].

$\mathrm{Al}$ respecto, el modelo logit replantea el modelo en términos de una función de distribución logística de la forma

\footnotetext{
${ }^{13}$ En ciudades fronterizas como Tijuana, Ciudad Juárez o Nogales, cerca de $2.5 \%$ de la población estudiantil en general, y $4 \%$ de los universitarios cruza cotidianamente para estudiar en EUA (Rocha Romero \& Orraca Romano, 2018).
} 


$$
P_{i}=\frac{1}{1+e^{-z_{i}}}=\frac{e^{z_{i}}}{1+e^{z_{i}}}
$$

donde, ahora, $Z_{i}=\alpha+\beta_{i} X_{i}+u$, y $P_{i}$ es la probabilidad de que el suceso ocurra, por lo que la probabilidad de que este no ocurra es $1-P_{i}$, que también puede ser representada de la siguiente manera:

$$
1-P_{i}=\frac{1}{1+e^{z_{l}}}
$$

Por lo tanto:

$$
\frac{P_{i}}{1-P_{i}}=e^{z_{i}}
$$

La razón de probabilidad en favor de que el suceso ocurra con respecto a que no sea así es $P_{i} /\left(1-P_{i}\right)$. Al tomar el logaritmo de la ecuación anterior se tiene

$$
\ln \left(\frac{P_{i}}{1-P_{i}}\right)=\alpha+\beta_{i} X_{i}+u
$$

que finalmente es el modelo a estimar. Se destaca que el logaritmo de la razón de probabilidad es lineal en las $X$ y en los parámetros $\beta$, y que las $X$ dependerán de la especificación que se trate.

\section{Descripción de variables}

Las variables explicativas utilizadas fueron seleccionadas con base en Kandel y Kao (2000), Antman (2011) y Vargas Valle (2015). En total se incluyen 20 variables independientes: seis de índole demográfica y socioeducativa (VDSE), ocho referidas al ámbito migratorio (VAMI) y seis más, relacionadas con el empeño, la motivación propia y el apoyo familiar (VEMA). Si bien las categorías VDSE, VAMI y VEMA no son exhaustivas ni mutuamente excluyentes, y las variables que las componen son apenas proxies de las dimensiones teóricas que pretenden medir, ${ }^{14}$ permiten diferenciar la naturaleza de la formación de las aspiraciones. La Tabla 2 presenta la descripción de las variables explicativas, su categoría y la forma que pueden tomar las respuestas.

En el presente trabajo la variable dependiente, $Y$, está referida a la aspiración del individuo de asistir a la universidad; la respuesta $Y=0$, significa que dicho atributo no está presente, mientras que $Y=1$, indica su presencia.

\footnotetext{
${ }^{14}$ Por ejemplo, las variables "país con el que más se identifica" y "país dónde se ve trabajando al terminar la escuela" podrían parecer VAMI, pero se ha optado por dejar en esta categoría solo decisiones y hechos, e incluir las variables relacionadas con expectativas en VEMA. Por otra parte, la variable "ha asistido a una escuela en EUA" podría vincularse a VDSE, sin embargo, al constituir un fuerte nexo transfronterizo se ha colocado en VAMI.
} 
Tabla 2. Variables independientes utilizadas en el estudio de las aspiraciones educativas

\begin{tabular}{|c|c|c|}
\hline Categoría & Variable* & Respuestas posibles \\
\hline \multirow{6}{*}{ VDSE } & Años cumplidos & $14,15, \ldots, 19$ \\
\hline & Número de hermanos & $\begin{array}{l}\text { Ninguno, uno o dos, tres o } \\
\text { cuatro, cinco o más }\end{array}$ \\
\hline & $\begin{array}{l}\text { Tener hermanos que asistan o hayan asistido a la } \\
\text { universidad }\end{array}$ & $\begin{array}{l}\text { No tiene hermanos o no están } \\
\text { en edad de asistir, no, sí. }\end{array}$ \\
\hline & Nivel educativo de la madre & \multirow{2}{*}{$\begin{array}{l}\text { Básico incompleto, básico, } \\
\text { medio superior, superior }\end{array}$} \\
\hline & Nivel educativo del padre & \\
\hline & Nivel de inglés & Nulo, bajo, alto \\
\hline \multirow{8}{*}{ VAMI } & Lugar de nacimiento & \multirow{3}{*}{ México, EUA } \\
\hline & Lugar de nacimiento de la madre & \\
\hline & Lugar de nacimiento del padre & \\
\hline & Ha asistido a una escuela en EUA & \multirow{4}{*}{ No, sí } \\
\hline & Ha vivido en EUA & \\
\hline & Su hogar recibe remesas & \\
\hline & Cuenta con familiares cercanos en EUA & \\
\hline & Los padres viven en EUA & Ninguno, alguno, ambos. \\
\hline \multirow{6}{*}{ VEMA } & Horas de estudio a la semana fuera de la escuela & $2,3, \ldots, 12$ \\
\hline & País con el que más se identifica & Ninguno, EUA, México, ambos \\
\hline & País dónde se ve trabajando al terminar la escuela & EUA, ambos, México, otro país \\
\hline & $\begin{array}{l}\text { Los padres se aseguran del cumplimiento de las } \\
\text { tareas escolares }\end{array}$ & \multirow{3}{*}{$\begin{array}{l}\text { Muy de acuerdo, de acuer- } \\
\text { do, en desacuerdo, muy en } \\
\text { desacuerdo }\end{array}$} \\
\hline & $\begin{array}{l}\text { Grado de acuerdo de los padres sobre la asistencia } \\
\text { a la universidad }\end{array}$ & \\
\hline & Grado de acuerdo de los padres respecto a trabajar & \\
\hline
\end{tabular}

Fuente: Elaboración propia con datos del MMFRP 2015-2016.

${ }^{*}$ A menos que se indique otra cosa, las preguntas aluden al estudiante.

\section{Análisis de resultados}

En primer término, con apoyo de estadística deductiva se describe la población bajo estudio para, posteriormente, calcular la magnitud del efecto de las variables independientes sobre las aspiraciones de asistir a la universidad, por medio de estadística inferencial correspondiente al modelo logístico.

\section{Análisis estadístico descriptivo}

La Tabla 3 muestra la distribución porcentual de las variables independientes no cuantitativas que serán utilizadas en el análisis estadístico inferencial, en función de la variable dependiente, lo que permite hacer una caracterización de los 2004 jóvenes de Tijuana, diferenciados por su tipo de expectativa educativa. 
Tabla 3. Distribución de las variables independientes no cuantitativas con respecto a la variable dependiente (porcentajes) de los jóvenes residentes de Tijuana

\begin{tabular}{|c|c|c|c|c|c|}
\hline Categoría & Variable & Respuestas posibles & $\begin{array}{l}\text { No aspira a asistir a } \\
\text { la universidad (\%) }\end{array}$ & $\begin{array}{l}\text { Aspira a asistir a la } \\
\text { universidad }(\%)\end{array}$ & $\begin{array}{l}p \text { - valor } \\
\text { del es- } \\
\text { tadístico }\end{array}$ \\
\hline \multirow{14}{*}{ VDSE } & \multirow{3}{*}{$\begin{array}{l}\text { Tener her- } \\
\text { manos que } \\
\text { asistan o hayan } \\
\text { asistido a la } \\
\text { universidad }\end{array}$} & $\begin{array}{l}\text { No tiene hermanos o no } \\
\text { están en edad de asistir }\end{array}$ & 43.55 & 48.00 & \multirow{3}{*}{0.000} \\
\hline & & No & 34.68 & 18.10 & \\
\hline & & Sí & 21.77 & 33.90 & \\
\hline & \multirow{4}{*}{$\begin{array}{l}\text { Nivel educativo } \\
\text { de la madre }\end{array}$} & Básico incompleto & 33.93 & 20.61 & \multirow{4}{*}{0.009} \\
\hline & & Básico & 40.53 & 33.04 & \\
\hline & & Media superior & 19.37 & 31.36 & \\
\hline & & Superior & 6.17 & 14.99 & \\
\hline & \multirow{4}{*}{$\begin{array}{l}\text { Nivel educativo } \\
\text { del padre }\end{array}$} & Básico incompleto & 27.55 & 16.79 & \multirow{4}{*}{0.017} \\
\hline & & Básico & 39.79 & 31.28 & \\
\hline & & Medio superior & 26.02 & 33.50 & \\
\hline & & Superior & 6.65 & 18.43 & \\
\hline & \multirow{3}{*}{ Nivel de inglés } & Nulo & 50.80 & 51.75 & \multirow{3}{*}{0.000} \\
\hline & & Bajo & 44.00 & 33.53 & \\
\hline & & Alto & 5.20 & 14.72 & \\
\hline \multirow{2}{*}{ VAMI } & \multirow{2}{*}{$\begin{array}{l}\text { Su hogar recibe } \\
\text { remesas }\end{array}$} & Sí & 24.88 & 15.95 & \multirow{2}{*}{0.007} \\
\hline & & No & 75.12 & 84.05 & \\
\hline \multirow{20}{*}{ VEMA } & \multirow{4}{*}{$\begin{array}{l}\text { País con el que } \\
\text { más se identi- } \\
\text { fica }\end{array}$} & Ninguno & 13.55 & 14.00 & \multirow{4}{*}{0.044} \\
\hline & & EUA & 12.26 & 9.56 & \\
\hline & & México & 54.19 & 45.43 & \\
\hline & & Ambos & 20.00 & 31.01 & \\
\hline & \multirow{4}{*}{$\begin{array}{l}\text { País dónde se } \\
\text { ve trabajando } \\
\text { al terminar la } \\
\text { escuela }\end{array}$} & EUA & 18.06 & 15.93 & \multirow{4}{*}{0.029} \\
\hline & & Ambos & 21.29 & 30.18 & \\
\hline & & México & 52.90 & 31.98 & \\
\hline & & Otro país & 7.74 & 13.91 & \\
\hline & \multirow{4}{*}{$\begin{array}{l}\text { Los padres se } \\
\text { aseguran del } \\
\text { cumplimiento } \\
\text { de las tareas } \\
\text { escolares }\end{array}$} & Muy de acuerdo & 9.68 & 9.72 & \multirow{4}{*}{0.031} \\
\hline & & De acuerdo & 23.87 & 12.07 & \\
\hline & & En desacuerdo & 29.68 & 20.96 & \\
\hline & & Muy en desacuerdo & 36.77 & 57.25 & \\
\hline & \multirow{4}{*}{$\begin{array}{l}\text { Grado de } \\
\text { acuerdo de los } \\
\text { padres sobre la } \\
\text { asistencia a la } \\
\text { universidad }\end{array}$} & Muy de acuerdo & 34.19 & 64.96 & \multirow{4}{*}{0.000} \\
\hline & & De acuerdo & 50.97 & 31.27 & \\
\hline & & En desacuerdo & 10.97 & 2.01 & \\
\hline & & Muy en desacuerdo & 3.97 & 1.76 & \\
\hline & \multirow{4}{*}{$\begin{array}{l}\text { Grado de } \\
\text { acuerdo de los } \\
\text { padres respecto } \\
\text { a trabajar }\end{array}$} & Muy de acuerdo & 14.19 & 18.78 & \multirow{4}{*}{0.017} \\
\hline & & De acuerdo & 54.19 & 61.27 & \\
\hline & & En desacuerdo & 22.58 & 15.09 & \\
\hline & & Muy en desacuerdo & 9.03 & 4.86 & \\
\hline \multicolumn{3}{|c|}{ Número de observaciones } & 257 & 1747 & \\
\hline
\end{tabular}

Fuente: Elaboración propia con datos del MMFRP 2015-2016.

Nota: Los porcentajes corresponden a la frecuencia de cada respuesta en relación con el total por variable. 
De acuerdo con Giorguli Saucedo y colaboradores (2010), una familia numerosa puede influir en las intenciones de los jóvenes de asistir a la universidad. Al respecto, se observa en la Tabla 3 que tener más de dos hermanos es más frecuente en los casos en los que no se aspira a asistir a la universidad, aunque si estos han asistido a la universidad podrían constituir un ejemplo académico que, compensatoriamente, incremente el interés por estudiar.

También, se evidencia que el nivel educativo de los padres es mayor en la población estudiantil que pretende asistir a la universidad. Hasta $46 \%$ de las madres y $52 \%$ de los padres tenían al menos educación media superior cuando estaban presentes las intenciones de los hijos de hacer una licenciatura, contra $26 \%$ y $33 \%$, respectivamente, cuando no estaba presente este atributo. Conjuntamente, la proporción de jóvenes que tiene un nivel alto de inglés entre los que desean adquirir estudios universitarios es más del doble de la existente cuando no se tiene esta aspiración; en cualquier caso, el porcentaje no alcanza $15 \%$.

Es de resaltar que, debido a la distribución de las respuestas, muchas variables de índole migratoria, tales como que alguno de los padres haya nacido o vivido en EUA, contar con familiares en este país o el haber estudiado, vivido o nacido en él, a priori, no permiten individualmente segregar la intención de alcanzar estudios superiores. De cualquier forma, casi en todos los casos la población que sí aspira a asistir a la universidad tiene mayor vinculación con EUA, que los que no tienen este propósito, excepto en lo que se refiere a la recepción de remesas, la cual es más frecuente en las familias de jóvenes que no aspiran a asistir a la universidad. De hecho, las remesas suelen destinarse a comida, vestido, vivienda, salud y el pago de deudas, antes que a la educación (Fundación biva Bancomer, A. C. \& Consejo Nacional de Población [Conapo], 2016) y enfrentan retos complejos para poder llegar a convertirse en inversiones productivas (Durand et al., 1996).

Los que aspiran a asistir a la universidad se identifican menos con México y más con ambos países, que los que manifiestan no estar interesados en realizar estudios superiores. $\mathrm{Al}$ mismo tiempo, entre los que tienen intenciones universitarias, 32\% se ve trabajando en México y $30 \%$ en ambos países mientras que estos valores son de $53 \%$ y $21 \%$ para los que no las tienen. La necesidad del aseguramiento de las tareas escolares por parte de los padres es una acción que descarta la mayoría de los potenciales universitarios. Es interesante destacar también que, aunque existe mayor acuerdo de los padres en la asistencia a la universidad cuando existe este anhelo por el estudiante, también lo hay en el sentido de apoyar sus intereses laborales.

En lo referente a la visión acerca de la educación superior en la juventud tijuanense, al distinguir entre aquellos que tienen vinculación o experiencia transnacional (207, equivalente a $10 \%$ de los radicados en Tijuana) y los que no la tienen, se encuentra que no existe diferencia estadística en la importancia que dan ambos grupos a asistir a la universidad (66\% dan mucha importancia). Sin embargo, la población joven con vínculos transnacionales tiene más claridad en cuanto a la intención de asistir a una universidad estadounidense, ${ }^{15}$ lo que es pretendido por $70 \%$ de este subconjunto

${ }^{15}$ Esto ya había sido destacado por autores como Vargas Valle (2015) y Rocha Romero y Orraca Romano (2018). 
frente a $54 \%$ de los que no cumplen esta condición. Lo anterior es un indicio de la existencia de una relación entre las decisiones en materia de formación y el grado de vinculación transnacional que se tenga.

En cuanto al sentido de pertenencia, 23\% de los jóvenes con vínculos transnacionales se identifica más con la cultura de EuA que con la mexicana, mientras que esto solo ocurre en $8 \%$ de los que no tienen esta vinculación. Asimismo, $42 \%$ de estos jóvenes transnacionales se identifica con ambos países, lo que ocurre solo en $27 \%$ de los jóvenes no transnacionales. Es de destacar que este es un indicador con diferente ordenación jerárquica entre ambos colectivos. Mientras que los transnacionales se identifican en primer término con ambos países, después con México, posteriormente con eUA y finalmente con ninguno de los dos, en el caso de los no transnacionales el orden es México, ambos, ninguno y, por último, EuA.

\section{Análisis estadístico inferencial}

Con el objetivo de explorar la relación entre las variables independientes y la dependiente (las aspiraciones de educación superior de los jóvenes de Tijuana) se estimaron cuatro especificaciones del modelo logit, nombradas esp1, esp2, esp 3 y esp 4 , las cuales dibujan escenarios con distintas condiciones demográficas y socioeducativas (VDSE), migratorias (VAMI) y asociadas al empeño, motivación y apoyo familiar (VEMA) ${ }^{16}$ (ver Tabla 1).

Todas las especificaciones tienen su soporte en VDSE para conocer si, libre del efecto de la composición de las familias y su condición académica, el contexto migratorio o el empeño, motivación y apoyo de la familia son importantes. La segunda categoría más intensiva en el caso de esp1 y esp2 es VAMI, mientras que esp3 y esp 4 descansan significativamente en VEMA.

En términos de VAMI, la propuesta esp1 considera el origen de los individuos, la asistencia a escuelas en EUA, la recepción o no de remesas y el contar con familiares cercanos en EUA, en tanto que esp2, incluye la asistencia a escuelas en EUA y el lugar donde el estudiante ha vivido y aquel donde su familia nuclear vive o ha vivido. Por otra parte, las propuestas $e s p 3$ y esp 4 se diferencian en cuanto a las VEMA: $e s p 3$ incluye aspectos de empeño y motivación (las horas de dedicación a la escuela, el país con el que se identifica y aquel donde se ve trabajando en el futuro), mientras que esp4 incorpora las variables relacionadas con el apoyo de al menos alguno de los padres, con respecto al futuro académico y profesional de su hijo.

En cuanto a la significancia de los efectos marginales ${ }^{17}$ para las VDSE se destaca de la Tabla 4 que la edad resulta significativa y negativa, lo que da muestra de que el paso

\footnotetext{
${ }^{16}$ Por su contribución a la argumentación se mantienen las especificaciones originales, aun cuando contienen variables no significativas.

${ }^{17}$ Los efectos marginales representan la variación de la probabilidad cuando se incrementa en una unidad o nivel la variable predictora, mantiene el resto en su nivel medio
} 
del tiempo reduce los incentivos para hacer estudios superiores, acaso en parte por la mayor necesidad de contribuir con los ingresos económicos familiares (Zúñiga et al., 2008). También, el tener hermanos estudiando en la universidad, que el padre tenga estudios superiores y que la o el joven tenga un alto dominio del inglés, resultaron significativas y positivas en todas las especificaciones en que fueron incluidas, lo que llegó a incrementar las intenciones de hacer estudios superiores (medidas como probabilidad) en hasta $3.8 \%, 7.0 \%$ y $5.2 \%$, respectivamente. El nivel educativo de la madre resultó significativo solo en algunas pruebas y asociado mayoritariamente al nivel educativo medio superior (esp2 y esp3). ${ }^{18}$ Lo anterior podría ser producto de la cultura patriarcal, aun predominante, bajo la cual, por una parte, el padre sigue siendo la principal referencia de desarrollo profesional y, por la otra, la madre profesionista enfrenta los costos de oportunidad de la carga de trabajo no remunerado relacionada con la dedicación a los cuidados y la familia.

Con respecto a las VAMI, la recepción de remesas resultó significativa y con efectos negativos en las aspiraciones de asistir a la universidad. Sobre esto, estudios como los de Durand y colaboradores (1996), Giorguli y Serratos (2009) y Fundación bBva Bancomer, A. C. \& Conapo (2016) han identificado que la transformación de las remesas en inversiones productivas no es automática, se llega a encontrar que estas desincentivan la continuidad escolar debido al ajuste en la percepción del valor y costo de la educación ante la posibilidad de una migración laboral. ${ }^{19}$

En cuanto a las VEMA, en línea con los resultados de Antman (2011), cada hora de dedicación académica semanal fuera de la escuela incrementa en $1.4 \%$ las aspiraciones de realizar estudios universitarios. Sin embargo, las perspectivas acerca del país con el que la persona más se identifica o aquel en el que se visualiza desempeñando una profesión no parecen relacionarse significativamente con las intenciones académicas de acuerdo con las regresiones logit (lo cual contrasta con el resultado de la prueba chi-cuadrado de independencia para cada variable de la Tabla 3).

Finalmente, las tres variables que tienen que ver con el apoyo de los padres sobre esta decisión potencial de los jóvenes resultaron significativas, aunque de distinto signo. El asegurarse del cumplimiento de las $\operatorname{tareas}^{20} \mathrm{y}$ el acuerdo sobre la asistencia a la universidad tuvieron un efecto positivo en las aspiraciones (de 3.8\% y 6.2\%, respectivamente), mientras que el efecto de alentar planes distintos a los de estudiar, es negativo $(-6.2 \%)$. Lo anterior deja ver que el empeño y el respaldo recibido por la familia resultan relevantes en la conformación de las aspiraciones. Se debe notar que, aunque en el caso de los jóvenes con aspiraciones universitarias hay mayor conciliación entre su visión y la de sus padres, tanto en materia académica como laboral (ver Tabla 3), esta termina teniendo un efecto compensatorio por el cual el apoyo laboral contrarresta el impulso del apoyo académico.

\footnotetext{
${ }^{18}$ La falta de significancia de esta variable en esp1 podría deberse a un efecto compensatorio a través de variables no incluidas en el resto de las especificaciones.

${ }^{19}$ Se reitera que variables como el origen de los miembros de la familia, el hecho de haber vivido o estudiado en EUA o el que los padres u otros familiares vivieran en aquel país, no resultan determinantes para discernir entre los tipos de aspiraciones universitarias.

${ }^{20}$ Aunque por otra parte también es cierto que aquellos con aspiraciones de educación superior requieren menos de esta supervisión (ver Tabla 3).
} 
Tabla 4. Modelos de regresión logística para el estudio de las aspiraciones educativas (efectos marginales) de los jóvenes residentes de Tijuana

\begin{tabular}{|c|c|c|c|c|c|c|c|c|c|c|}
\hline \multirow{2}{*}{$\begin{array}{l}\text { Cate- } \\
\text { goría }\end{array}$} & \multirow{2}{*}{\multicolumn{2}{|c|}{ Variable }} & \multicolumn{8}{|c|}{ Coeficiente (Error estándar) } \\
\hline & & & \multicolumn{2}{|c|}{ esp1 } & \multirow{2}{*}{\multicolumn{2}{|c|}{$\frac{e s p 2}{-}$}} & \multirow{2}{*}{\multicolumn{2}{|c|}{$\frac{e s p 3}{-}$}} & \multirow{2}{*}{\multicolumn{2}{|c|}{$\begin{array}{c}\text { esp4 } \\
-\end{array}$}} \\
\hline \multirow{14}{*}{ VDSE } & \multicolumn{2}{|l|}{ Años cumplidos } & $-0.0264 * * *$ & $(0.009)$ & & & & & & \\
\hline & \multirow{3}{*}{$\begin{array}{l}\text { Número de } \\
\text { hermanos }\end{array}$} & Uno o dos & 0.0837 & $(0.059)$ & 0.0858 & $(0.059)$ & 0.0980 & $(0.060)$ & 0.0837 & $(0.058)$ \\
\hline & & Tres o cuatro & 0.0512 & $(0.038)$ & 0.0504 & $(0.039)$ & $0.0595^{*}$ & $(0.035)$ & 0.0494 & $(0.037)$ \\
\hline & & Cinco o más & 0.0491 & $(0.035)$ & 0.0482 & $(0.037)$ & 0.0431 & $(0.035)$ & 0.0495 & $(0.032)$ \\
\hline & \multirow{2}{*}{$\begin{array}{l}\text { Hermanos en } \\
\text { universidad }\end{array}$} & No & -0.0229 & $(0.021)$ & -0.0283 & $(0.022)$ & \multicolumn{2}{|l|}{-} & -0.0258 & $(0.021)$ \\
\hline & & Si & $0.0383 * *$ & $(0.018)$ & $0.0376 * *$ & $(0.018)$ & \multicolumn{2}{|l|}{ - } & $0.0353 * *$ & $(0.017)$ \\
\hline & \multirow{3}{*}{$\begin{array}{l}\text { Nivel educativo de } \\
\text { la madre }\end{array}$} & Básico & -0.0030 & $(0.019)$ & 0.0026 & $(0.019)$ & 0.0041 & $(0.018)$ & 0.0017 & $(0.019)$ \\
\hline & & Medio superior & 0.0311 & $(0.020)$ & $0.0354 *$ & $(0.020)$ & $0.0407 * *$ & $(0.019)$ & 0.0303 & $(0.020)$ \\
\hline & & Superior & 0.0307 & $(0.027)$ & 0.0373 & $(0.026)$ & 0.0348 & $(0.025)$ & $0.0400 *$ & $(0.024)$ \\
\hline & \multirow{3}{*}{$\begin{array}{l}\text { Nivel educativo del } \\
\text { padre }\end{array}$} & Básico & 0.0109 & $(0.019)$ & 0.0118 & $(0.019)$ & 0.0222 & $(0.018)$ & 0.0141 & $(0.018)$ \\
\hline & & Medio superior & 0.0269 & $(0.019)$ & 0.0279 & $(0.020)$ & $0.0360 *$ & $(0.018)$ & 0.0280 & $(0.019)$ \\
\hline & & Superior & $0.0453 * * *$ & $(0.023)$ & $0.0499 * *$ & $(0.023)$ & $0.0522 * *$ & $(0.021)$ & $0.0435^{*}$ & $(0.023)$ \\
\hline & \multirow{2}{*}{ Nivel de inglés } & Bajo & $0.0329 * *$ & $(0.016)$ & $0.0358 * *$ & $(0.017)$ & 0.0223 & $(0.016)$ & $0.0336^{* *}$ & $(0.016)$ \\
\hline & & Alto & $0.0610^{* * *}$ & $(0.020)$ & $0.0700 * * *$ & $(0.019)$ & $0.0573^{* * *}$ & $(0.020)$ & $0.0594 * * *$ & $(0.019)$ \\
\hline \multirow{9}{*}{ VAMI } & \multicolumn{2}{|c|}{ Lugar de nacimiento (EUA) } & -0.0033 & $(0.051)$ & \multicolumn{2}{|l|}{-} & 0.0005 & $(0.048)$ & \multicolumn{2}{|l|}{-} \\
\hline & \multicolumn{2}{|c|}{ Lugar de nacimiento de la madre (EUA) } & -0.0223 & $(0.116)$ & \multicolumn{2}{|c|}{-} & 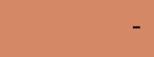 & & \multicolumn{2}{|l|}{-} \\
\hline & \multicolumn{2}{|c|}{ Lugar de nacimiento del padre (EUA) } & 0.0322 & $(0.046)$ & \multicolumn{2}{|c|}{ - } & - & & - & \\
\hline & Ha asistido a una esc & tela en EUA & -0.0420 & $(0.050)$ & -0.0024 & $(0.050)$ & -0.053 & $(0.053)$ & -0.0418 & $(0.048)$ \\
\hline & Ha vivido en EUA & & - & & -0.0587 & $(0.052)$ & - & & - & \\
\hline & Su hogar recibe rem & & $-0.0618 * *$ & $(0.026)$ & - & & - & & - & \\
\hline & Cuenta con familiare & cercanos en EUA & 0.0052 & $(0.024)$ & - & & - & & - & \\
\hline & Los padres viven & Alguno & - & & 0.0036 & $(0.032)$ & - & & - & \\
\hline & en EUA & Ambos & - & & -0.1564 & $(0.178)$ & - & & - & \\
\hline & Horas estudio fuera & e escuela & - & & - & & $0.0141 * * *$ & $(0.003)$ & - & \\
\hline & & EUA & - & & - & & -0.0324 & $(0.037)$ & - & \\
\hline & $\begin{array}{l}\text { Pais con el que mas } \\
\text { se identifica }\end{array}$ & México & - & & - & & 0.0021 & $(0.022)$ & - & \\
\hline & & Ambos & - & & - & & 0.0234 & $(0.023)$ & - & \\
\hline VEMA & País trabajando al & Ambos & - & & - & & 0.0172 & $(0.020)$ & - & \\
\hline & terminar escuela & México & - & & - & & -0.0283 & $(0.019)$ & - & \\
\hline & Padres aseguran tare & s escolares & - & & - & & - & & $0.0381 * *$ & $(0.016)$ \\
\hline & Acuerdo padres univ & rsidad & - & & - & & - & & $0.0622 * * *$ & $(0.019)$ \\
\hline & Acuerdo padres trab & & - & & - & & - & & $-0.0623 * *$ & $(0.027)$ \\
\hline & Número de obs & rvaciones & 200 & & 20 & & 200 & & 200 & \\
\hline & $\mathrm{R}^{2}$ de $\mathrm{McFa}$ & den & 0.07 & & 0.0 & & 0.08 & & 0.08 & \\
\hline & L.R. Chi-cua & drado & 71.6 & & 58. & & 82.0 & & 85.1 & \\
\hline
\end{tabular}

Fuente: Elaboración propia con datos del MMFRP 2015-2016.

Nota: Las variables cualitativas fueron tratadas como variables ficticias. Para evitar problemas de colinealidad las primeras opciones de las variables cualitativas (ver Tabla 1) fueron tomadas como base.

Se presentan los efectos marginales en lugar de los coeficientes. Significancia a ${ }^{*} 10 \%,{ }^{\star *} 5 \%$ y ${ }^{* *} 1 \%$. 
$\mathrm{Al}$ comparar el $R^{2}$ de Mcfadden de las regresiones se observa que el modelo esp4, conformado principalmente de variables demográficas, socioeducativas y asociadas al apoyo familiar es el que mejor explica el comportamiento de la variable dependiente. Si bien en todos los casos este indicador es relativamente bajo, también es cierto que la medida convencional de la bondad de ajuste no suele ser especialmente alta en las regresiones binarias (Gujarati \& Porter, 2010). Una segunda medida de ajuste, la prueba chi-cuadrado, permite corroborar que la especificación esp 4 es la más adecuada al arrojar el valor más alto (Tabla 4 ).

\section{Conclusiones}

Este trabajo buscó conocer si las aspiraciones de los jóvenes de educación media superior que radican en Tijuana, Baja California son más sensibles a la exposición al entorno migratorio que al empeño, la motivación y el respaldo recibido por la familia, al tomar en cuenta su condición de frontera internacional con San Diego en California.

Salvo en el caso de la recepción de remesas, las aspiraciones resultaron poco sensibles a muchas de las circunstancias migratorias y transnacionales. Al mismo tiempo, los anhelos académicos de los jóvenes dependen de sus estudios previos, tener hermanos que hayan asistido a la universidad y el nivel educativo de sus padres. Además, estas aspiraciones son altamente sensibles al apoyo y soporte familiar por parte de los padres, así como al empeño del joven.

De esta forma, aunque la evidencia muestra una relación negativa entre la exposición al contexto migratorio y transfronterizo (por ejemplo, el lugar de nacimiento y residencia de los padres o la experiencia académica en EUA) y las aspiraciones de estudios superiores, al no ser significativa, no es posible aceptar la hipótesis planteada en esta investigación. Sin bien es cierto que el resultado anterior podría estar relacionado con las limitaciones en el tamaño de la muestra, la especificidad del rango de edad de la población estudiada y el uso de una definición acotada del transfronterismo, también da muestra de que las características socioeducativas, la composición familiar y el soporte que esta pueda constituir tienen un peso importante en las decisiones sobre educación fronteriza.

Un reto destacable para el progreso de la ciudad de Tijuana, generalizable al de otras situadas en la frontera norte de México, es el de superar el rezago educativo de sus jóvenes. ${ }^{21}$ La posibilidad de migrar en busca de opciones de empleo, la alta oferta de trabajo de baja calificación en su país de residencia, sumado a problemas socioeconómicos como la desigualdad, la marginación y el desarraigo social hacen que no sea sencillo construir comunidades con proyección académica sólida en aquellos grupos de edad en los que la posibilidad de trabajar o de migrar empieza a ser considerada. Las autoridades en las zonas fronterizas deben abordar el reto del diseño e implementación de políticas públicas, con acciones afirmativas dirigidas a la juventud con nexos transfronterizos, en las que también sea incorporada la juventud migrante retornante y la proveniente de otras regiones (tanto del país como del extranjero), con miras a la integración y asentamiento de sus familias, de tal forma que puedan resarcirse los rezagos educativos y sociales y fortalecerse el capital humano y el tejido social, para el aprovechamiento de este bono demográfico.

${ }^{21}$ Asociada a priori con la desintegración familiar que puede llegar a ser generada por la migración. 
Las instituciones educativas deben incorporar en sus planes de estudios el perfil del capital humano que se requiere en la nueva economía local a partir de un esquema de economía globalizada. A la ecuación habría que incorporar también la visión de los jóvenes y de sus familias acerca del desarrollo local de la frontera y sobre la vecindad entre los dos países y, por consecuencia, de "lo transfronterizo" (Ojeda, 2009). Las ideas de Ganster (1994) siguen teniendo vigencia, en el sentido de la importancia de que las universidades evalúen las necesidades futuras de los sectores público, privado, social y académico, y que precisen los ajustes que requieren sus programas de estudio a fin de que los jóvenes profesionistas cuenten con las habilidades necesarias para desenvolverse en esta cambiante región binacional.

Sin duda, dada su heterogeneidad tanto desde el punto de vista ideológico como socioeconómico, se requiere más investigación sobre las comunidades estudiantiles con nexos transfronterizos para poder conocer los determinantes de sus expectativas y decisiones. Por ejemplo, tal como lo puntualizan Orraca y colaboradores (2017), no queda claro si después de la educación media superior los estudiantes con vínculos transfronterizos, que en su momento manifestaron aspiraciones de alcanzar la educación profesional, continuaron efectivamente estudiando, y en qué país lo hicieron.

\section{Referencias}

Aguilar Barceló, J. G., Mungaray Moctezuma, A. B., Jaramillo Cardona, M. C. \& Aguilar Solís, G. A. (2020). Migración y educación superior: expectativas de los jóvenes de la frontera Tijuana-San Diego. En J. G. Aguilar Barceló, A. B. Mungaray Moctezuma, M. C. Jaramillo Cardona \& S. López Leyva (Coords.), Innovación social, políticas públicas e instituciones para el desarrollo de las regiones (pp. 151-172). UABC/ Ediciones del Lirio.

Antman, F. M. (2011). The intergenerational effects of paternal migration on schooling and work: what can we learn from children's time allocations? Journal of Development Economics, 96(2), 200-208. https://doi.org/10.1016/j.jdeveco.2010.11.002

Becker, G. S. (1964). Human capital: a theoretical and empirical analysis, with special reference to education. The University of Chicago Press.

Booth, M. Z. (1995). Children of migrant fathers: the effects of father absence on Swazi children's preparedness for school. Comparative Education Review, 39(2), 195-210. http:/ / www.jstor.org/stable/1188928

Borraz, F. (2005). Assessing the impact of remittances on schooling: the Mexican experience. Global Economy Journal, 5(1), 1-30. https://doi.org/10.2202/15245861.1054

Calderón, C., Díaz, E., Mendoza, E. \& Hernández, L. (2010). El desempleo en los estados de la frontera norte de México. Documento de Coyuntura. El Colegio de la Frontera Norte.

Canales, A. (2012). La migración mexicana frente a la crisis económica actual. Crónica de un retorno moderado. Revista Interdisciplinar da Mobilidade Humana, 20(39), 117-134. https://www.scielo.br/j/remhu/a/FB8WWkdHsgmGjpNdRkJ8Q4x/ abstract/?lang=es\&format=html\# 
Coubès, M.-L. \& González Ramírez, R. S. (2011). Experiencias de vida de los jóvenes en Tijuana: las interrelaciones entre escuela y trabajo. En N. Ojeda de la Peña \& M. E. Zavala-Cosío (Coords.), Jóvenes fronterizos/border youth: expectativas de vida familiar, educación y trabajo hacia la adultez (pp. 56-76). El Colegio de la Frontera Norte/Conacyt.

Cruz Piñeiro, R. (2012). Cambios fronterizos y movimientos migratorios en la frontera norte de México. En T. Ramírez García \& M. A. Castillo (Coords.), El estado de la migración. México ante los recientes desafíos de la migración internacional (pp. 157184). Consejo Nacional de Población. https://imumi.org/attachments/mexico_recientes_desafios.pdf

Durand, J., Kandel, W., Parrado, E. A. \& Massey, D. S. (1996). International migration and development in Mexican communities. Demography, 33(2), 249-264. https://doi.org/10.2307/2061875

El Colegio de la Frontera Norte (2019). La caravana de migrantes centroamericanos en Tijuana 2018-2019 (segunda etapa). El Colegio de la Frontera Norte. https:// www.colef.mx/wp-content/uploads/2019/03/2o.-Reporte-Caravana-Tijuana.250319.pdf

Espitia Carrascal, R. E. \& Montes Rotela, M. (2009). Influencia de la familia en el proceso educativo de los menores del barrio Costa Azul de Sincelejo (Colombia). Investigación y Desarrollo, 17(1), 84-105. http://www.scielo.org.co/pdf/indes/ v17n1/v17n1a04.pdf

Fischer, P. A., Martin, R. \& Straubhaar T. (1997). Interdependencies between development and migration. En T. Hammar, G. Brochmann, K. Tamas \& T. Faist (Eds.), International migration, immobility and development. Multidisciplinary perspectives (pp. 91-132). Berg Publishers.

Fundación вbva Bancomer, A. C. \& Consejo Nacional de Población (Conapo). (2016). Anuario de migración y remesas. México 2016. https://www.gob.mx/cms/uploads/ attachment/file/109457/Anuario_Migracion_y_Remesas_2016.pdf

Ganster, P. (1994). La educación superior en la frontera Estados Unidos-México ante el TLC. Comercio Exterior, 44, 242-248. http://revistas.bancomext.gob.mx/rce/ magazines/357/9/RCE9.pdf

Giorguli, S. E. \& Serratos, I. (2009). El impacto de la migración internacional sobre la asistencia escolar en México: ¿paradojas de la migración? En P. Leite \& S. E. Giorguli (Coords.), El estado de la migración. Las políticas públicas ante los retos de la migración mexicana a Estados Unidos (pp. 313-344). Consejo Nacional de Población.

Giorguli Saucedo, S. E., Vargas Valle, E. D., Salinas Ulloa, V., Hubert, C. \& Potter, J. E. (2010). La dinámica demográfica y la desigualdad educativa en México. Estudios Demográficos y Urbanos, 25(73), 7-44. https://doi.org/10.24201/edu.v25i1.1366

Glick Schiller, N., Basch, L. \& Blanc-Szanton, C. (1992). Transnationalism: a new analytic framework for understanding migration. Annals of the New York academy of sciences, 645(1), 1-24. https://doi.org/10.1111/j.1749-6632.1992.tb33484.x

Glick Schiller, N., Basch, L. \& Blanc, C. S. (1995). From immigrant to transmigrant: theorizing transnational migration. Anthropological Quarterly, 86(1), 48-63. https://doi.org/10.2307/3317464 
Goldscheider, C. (1987). Migration and social structure: analytic issues and comparative perspectives in developing nations. Sociological Forum, 2(4), 674-696. https://www.jstor.org/stable/684298

González Galbán, H. (2011). Caracterización sociodemográfica de la población en tránsito a la vida adulta en Tijuana. En N. Ojeda de la Peña \& M. E. Zavala-Cosío (Coords.), Jóvenes fronterizos/border youth: expectativas de vida familiar, educación y trabajo hacia la adultez (pp. 23-56). El Colegio de la Frontera Norte/Conacyt.

Gujarati, D. \& Porter, D. (2010). Econometría. McGraw-Hill.

Hanson, G. H. \& Woodruff, C. (2003). Emigration and educational attainment in Mexico. Working Paper, University of California San Diego/National Bureau of Economics Research. https://citeseerx.ist.psu.edu/viewdoc/download?doi $=10.1 .1 .716 .5969 \&$ rep=rep1\&type $=$ pdf

Instituto Nacional de Estadística y Geografía (Inegi). (2010). Censo de población y vivienda 2010. https://inegi.org.mx/programas/ccpv/2010/

Instituto Nacional de Estadística y Geografía (Inegi). (2015). Encuesta intercensal 2015. https://www.inegi.org.mx/programas/intercensal/2015/

Instituto Nacional de Estadística y Geografía (Inegi). (2020). Censo de población y vivienda 2020. https://inegi.org.mx/programas/ccpv/2020/

Izquierdo Escribano, A. (2011). Times of losses: a false awareness of the integration of immigrants, Migraciones Internacionales, 6(20), 145-184. https://doi. org/10.17428/rmi.v6i20.1063

Kandel, W. \& Kao, G. (2000). Shifting orientations: how U.S. labor migration affects children's aspirations in Mexican migrant communities. Social Science Quarterly, 81(1), 16-32. https://www.jstor.org/stable/42864365

Kandel, W. \& Massey, D. S. (2002). The culture of Mexican migration: a theoretical and empirical analysis. Social Forces, 80(3), 981-1004. https://doi.org/10.1353/ sof.2002.0009

Leyva López, S. \& Cárdenas Almagro, A. (2002). Economía de la educación: capital humano y rendimiento educativo. Análisis Económico, 17(36), 79-106. https:// www.redalyc.org/pdf/413/41303603.pdf

Lindstrom, D. P. (1996). Economic opportunity in Mexico and return migration from the United States. Demography, 33(3), 357-374. https://www.jstor.org/stable $/ 2061767$

Marmolejo, F. \& León, F. (2000). La educación superior en la frontera México-Estados Unidos: convergencias y divergencias. Revista de la Educación Superior, 29(115), $1-19$.

Masferrer, C. \& Roberts, B. R. (2012). Going back home? Changing demography and geography of Mexican return migration. Population Research and Policy Review, 31, 465-496. https://doi.org/10.1007/s11113-012-9243-8

Massey, D. S. \& Parrado, E. A. (1998). International migration and business formation in Mexico, Social Science Quarterly, 79(1), 1-20. https://www.jstor.org/stable/42863761

McConnell, C. R., Brue, S. L. \& Macpherson, D. A. (2007). Economía laboral. McGraw-Hill.

McKenzie, D. \& Rapoport, H. (2011). Can migration reduce educational attainment? Evidence from Mexico. Journal of Population Economics, 24, 1331-1358. https:// doi.org/10.1007/s00148-010-0316-x 
Mendoza Cota, J. E. (2013). Migración de retorno, niveles educativos y desarrollo socioeconómico regional de México. Estudios Sociales, 21(42), 55-85. http://ref. scielo.org/nyybqq

Meza González, L. \& Pederzini Villarreal, C. (2009). Migración internacional y escolaridad como medios alternativos de movilidad social: el caso de México. Estudios Económicos, (Número extraordinario), 163-206. https://estudioseconomicos. colmex.mx/index.php/economicos/article/view/385/464

Münch, L. (2005). Administración de capital humano, la gestión del activo más valioso de la organización. Editorial Trillas.

Nobles, J. (2011). Parenting from abroad: migration, nonresident father involvement, and children's education in Mexico. Journal of Marriage and Family, 73(4), 729746. https://doi.org/10.1111/j.1741-3737.2011.00842.x

Ojeda, N. (2009). Reflexiones acerca de las familias transfronterizas y las familias transnacionales entre México y Estados Unidos. Frontera Norte, 21(42), 7-30. https:// doi.org/10.17428/rfn.v21i42.962

Orraca, P., Rocha, D. \& Vargas, E. (2017). Cross-border school enrolment: associated factors in the U.S.-Mexico borderlands, The Social Science Journal, 54(4), 389-402. https://doi.org/10.1016/j.soscij.2017.07.008

Rocha Romero, D. \& Orraca Romano, P. (2018). Estudiantes de educación superior transfronterizos: residir en México y estudiar en Estados Unidos. Frontera Norte, 30(59), 103-128. https://doi.org/10.17428/rfn.v30i59.880

Sarabia, H. (2015) Global South cosmopolitans: the opening and closing of the usa-Mexico border for Mexican tourists. Ethnic and Racial Studies, 38(2), 227242, https://doi.org/10.1080/01419870.2014.887741

Sistema Educativo Estatal de Baja California (sеEвC). (2018). Principales cifras estadísticas. Anuario de datos e indicadores educativos. Ciclo escolar 2017-2018. Secretaría de Educación. http://www.educacionbc.edu.mx/publicaciones/estadisticas/2018/publicaciones/Cuadernillo\%202017-2018.pdf

Taylor, J. E. (1987). Undocumented Mexico-US migration and the returns to households in rural Mexico. American Journal of Agricultural Economics, 69(3), 626-638. https://doi.org/10.2307/1241697

Vargas-Valle, E. D. (2012). Transborder links and formal education of urban youth on the Northern Border. Frontera Norte, 24(47), 7-30. https://doi.org/10.17428/ rfn.v24i47.813

Vargas Valle, E. D. (2015). A decade of changes: formal education and transborder linkages of young people in highly urbanized areas of the northern border. Estudios Fronterizos, 16(32), 1-14. http://dx.doi.org/10.21670/ref.2015.32.a05

Vargas-Valle, E. D. \& Glick, J. E. (2021). Educational and migration aspirations among children of Mexican migrant returnees in a border context. Migration Studies, 9(3), 677-701. https://doi.org/10.1093/migration/mnab014

Zúñiga, V., Hamann, E. T. \& Sánchez García, J. (2008). Alumnos transnacionales. Escuelas mexicanas frente a la globalización. Secretaría de Educación Pública.

Zúñiga, V. \& Reyes, M. (2006). La cultura de los pasaporteados: familia y migración internacional en Vallecillo, Nuevo León. En I. Ortega (Coord.), El Noreste. Reflexiones, (pp. 105-126). Fondo Editorial de Nuevo León. 
José G. Aguilar Barceló

Mexicano. Doctor en ciencias económicas por la UABc. Actualmente es director general en la Unidad de Desarrollo Productivo de la Secretaría de Economía. Profesor-Investigador (con licencia) de la Facultad de Economía y Relaciones Internacionales de la UABC. Especializado en análisis de la competencia, economía del desarrollo, desigualdad, pobreza y empresarialidad de base social. Es miembro del Sistema Nacional de Investigadores, nivel II, y de la Academia Mexicana de Ciencias. Publicación reciente: Aguilar Barceló, J. G. \& Acuña Garcés, R. A. (2021). Inclusión de la propensión al autoempleo en el proceso de emparejamiento del mercado laboral. Problemas del Desarrollo, 52(207), 107-131. https://doi.org/10.22201/ iiec.20078951e.2021.207.69741

Germán Osorio-Novela

Mexicano. Doctor en ciencias económicas por la UABC. Subdirector de la Facultad de Economía y Relaciones Internacionales de la UABC. Líneas de investigación: emprendimiento, economía de la microempresa y negocios sociales. Publicación reciente: Mungaray, A., González Arzabal, N. \& Osorio Novela, G. (2021). Educación financiera y su efecto en el ingreso en México. Problemas del Desarrollo, 52(205). https:/ / doi.org/10.22201/iiec.20078951e.2021.205.69709

Guillermo A. Aguilar Solís

Mexicano. Maestro en ciencias económicas por la UABC. Actualmente estudiante del doctorado en estudios de migración de El Colef. Líneas de investigación: selectividad de la trayectoria exitosa de los centroamericanos en tránsito por México hacia Estados Unidos. Publicación reciente: Aguilar-Barceló, J. G., Mungaray-Moctezuma, A. B., Jaramillo Cardona, M. C. \& Aguilar Solís, G. A. (2020). Migración y educación superior: expectativas de los jóvenes de la frontera Tijuana-San Diego. En J. G. Aguilar Barceló, A. B. Mungaray Moctezuma, M. C. Jaramillo Cardona \& S. López Leyva (Coords.), Innovación social, políticas públicas e instituciones para el desarrollo de las regiones (pp. 151172). UABC/Ediciones del Lirio.

Ana B. Mungaray-Moctezuma

Mexicana. Doctora en ciencias económicas por la UABC. Cuenta con el reconocimiento al perfil deseable Prodep y es miembro del Sistema Nacional de Investigadores, nivel I. Es jefa de la Unidad de Desarrollo Productivo en la Secretaría de Economía. Sus áreas de investigación son el fomento de Mipymes y la economía política del desarrollo. Publicaciones recientes: Pérez Núñez, S. M. \& Mungaray Moctezuma, A. B. (2018). Capacidades de innovación de las bioempresas acuícolas de Baja California. En J. M. Ocegueda \& A. Mungaray (Coords.), Lento crecimiento y caída del bienestar en la economía de Baja California (173-198). UABC. 